\title{
Gamma-Band Activity in Human Prefrontal Cortex Codes for the Number of Relevant Items Maintained in Working Memory
}

\author{
Frédéric Roux, ${ }^{1,2}$ Michael Wibral, ${ }^{1}$ Harald M. Mohr ${ }^{3,4}$ Wolf Singer, ${ }^{2,5,6}$ and Peter J. Uhlhaas ${ }^{2,6}$ \\ ${ }^{1}$ MEG Unit, Brain Imaging Center, Johann Wolfgang Goethe University, 60528 Frankfurt am Main, Germany, ${ }^{2}$ Department of Neurophysiology, Max-Planck \\ Institute for Brain Research, 60528 Frankfurt am Main, Germany, ${ }^{3}$ Department of Neurocognitive Psychology, Institute of Psychology, Johann Wolfgang \\ Goethe University, 60325 Frankfurt am Main, Germany, ${ }^{4}$ Department of Psychiatry, Psychosomatic Medicine and Psychotherapy, Johann Wolfgang Goethe \\ University, 60528 Frankfurt am Main, Germany, ${ }^{5}$ Frankfurt Institute for Advanced Studies, Johann Wolfgang Goethe University, 60438 Frankfurt am Main, \\ Germany, and ${ }^{6}$ Ernst-Strüngmann Institute (ESI) for Neuroscience in cooperation with the Max-Planck-Society, 60528 Frankfurt am Main, Germany
}

Previous studies in electrophysiology have provided consistent evidence for a relationship between neural oscillations in different frequency bands and the maintenance of information in working memory (WM). While the amplitude and cross-frequency coupling of neural oscillations have been shown to be modulated by the number of items retained during WM, interareal phase synchronization has been associated with the integration of distributed activity during WM maintenance. Together, these findings provided important insights into the oscillatory dynamics of cortical networks during WM. However, little is known about the cortical regions and frequencies that underlie the specific maintenance of behaviorally relevant information in WM. In the current study, we addressed this question with magnetoencephalography and a delayed match-to-sample task involving distractors in 25 human participants. Using spectral analysis and beamforming, we found a WM load-related increase in the gamma band $(60-80 \mathrm{~Hz})$ that was localized to the right intraparietal lobule and left Brodmann area 9 (BA9). WM-load related changes were also detected at alpha frequencies $(10-14 \mathrm{~Hz})$ in Brodmann area 6, but did not covary with the number of relevant WM-items. Finally, we decoded gamma-band source activity with a linear discriminant analysis and found that gamma-band activity in left BA9 predicted the number of target items maintained in WM. While the present data show that WM maintenance involves activity in the alpha and gamma band, our results highlight the specific contribution of gamma band delay activity in prefrontal cortex for the maintenance of behaviorally relevant items.

\section{Introduction}

Working memory (WM) is a fundamental cognitive function that permits the maintenance of sensory information over a short period of time (Baddeley, 2003). Theoretical work suggests that temporally correlated activity in recurrent cell assemblies may sustain sensory information in the absence of external input (Hebb, 1949). In addition, synchronous fluctuations of neural activity have been suggested to bind the activity of cortical cell assemblies (Buzsáki and Draguhn, 2004). Accordingly, neural oscillations have been proposed as a mechanism to maintain the neural representations of sensory information during WM (Jensen et al., 2007).

\footnotetext{
Received Jan. 30, 2012; revised June 11, 2012; accepted July 5, 2012

Author contributions: F.R., H.M.M., and P.J.U. designed research; F.R. performed research; F.R., M.W., and P.J.U. analyzed data; F.R., M.W., W.S., and P.J.U. wrote the paper.

This work was supported by the Max-Planck Society and the LOEWE Grant "Neuronale Koordination Forschungsschwerpunkt Frankfurt."

This article is freely available online through the J Neurosci Open Choice option.

The authors declare no competing financial interests.

Correspondence should be addressed to Dr. Peter J. Uhlhaas, Department of Neurophysiology, Max-Planck Institute for Brain Research, Deutschordenstrasse 46, 60528 Frankfurt am Main, Germany. E-mail: peter.uhlhaas@brain.mpg.de.

DOI:10.1523/JNEUROSCI.0421-12.2012

Copyright $\odot 2012$ the authors $\quad 0270-6474 / 12 / 3212411-10 \$ 15.00 / 0$
}

Support for this hypothesis was first provided by WM studies in humans (Tallon-Baudry et al., 1998) and nonhuman primates (Pesaran et al., 2002), which reported a sustained increase of oscillatory activity in the gamma frequency range during the memory period of delayed response tasks. Subsequently, a large body of evidence has documented a parametric relationship between the amplitude of oscillatory activity at theta, alpha, beta, and gamma frequencies and the number of WM items memorized during memory-scanning tasks (Jensen and Tesche, 2002; Howard et al., 2003; Onton et al., 2005; Meltzer et al., 2008), visuospatial WM (Busch and Herrmann, 2003; Sauseng et al., 2005, 2009; Palva et al., 2011), and auditory WM (Leiberg et al., 2006).

Delay activity in the theta band (Jensen and Tesche, 2002; Meltzer et al., 2008) and the gamma band (Howard et al., 2003; Meltzer et al., 2008; Palva et al., 2011) have both been associated with the active maintenance of sensory representations during WM. In contrast, modulations of spectral power at alpha frequencies have been assigned different roles. While some studies have related delay activity in the alpha band with the functional inhibition of task-irrelevant brain areas (Jensen et al., 2002; Jokisch and Jensen, 2007; Medendorp et al., 2007; Sauseng et al., 2009; Van Der Werf et al., 2010), others have linked alpha- 
a

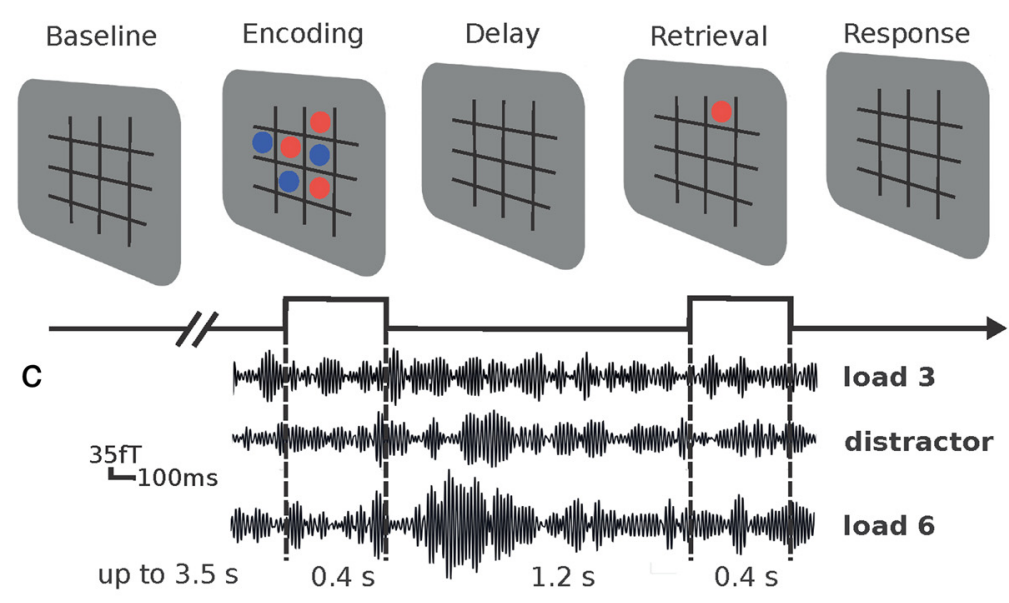

b

Load 3

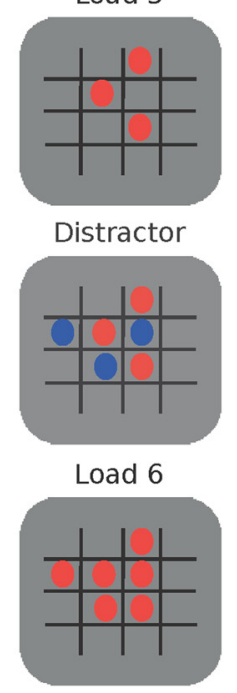

Figure 1. The visuospatial working memory task. $a$, Example of a trial with distractors during the encoding. On one-third of the trials, participants were shown three red discs together with three blue discs (distractors), and participants were asked to memorize the positions of the red discs only and to ignore the positions of the blue discs. In the remaining trials, distractors were absent and either three or six red discs were presented. After a maintenance phase of $1.2 \mathrm{~s}$, a test item was presented at a position identical (match) or different (nonmatch) to the sample array. $\boldsymbol{b}$, Example of the memory arrays for the three conditions: load 3, distractor, and load 6. c, Sample traces of bandpass ( 60 - $80 \mathrm{~Hz}$ )-filtered MEG signals during the load 3, distractor, and load 6 conditions from one single subject. The recording comprises the baseline, encoding, delay, and retrieval periods. Note the enhanced oscillations in the gamma frequency in the delay phase of the load 6 condition.

band activity directly to processes underlying WM maintenance (Busch and Herrmann, 2003; Herrmann et al., 2004; Sauseng et al., 2005; Leiberg et al., 2006; Palva et al., 2011). In addition to the contribution of individual frequency bands, theoretical and empirical studies have implicated the coupling between oscillation frequencies as a mechanism for WM maintenance. Specifically, theta-nested gamma-band oscillations have been proposed to sequentially code the order of WM items (Lisman and Idiart, 1995), which is supported by intracranial EEG recordings (Axmacher et al., 2010) as well as by scalp-recorded EEG/MEG data (Sauseng et al., 2009; Fuentemilla et al., 2010).

More recently, EEG and MEG studies have mapped the oscillatory networks during WM retention. In these studies, gammaband delay activity was localized to core regions of the WM network, previously identified by functional magnetic resonance imaging (fMRI) (Wager and Smith, 2003), including occipital, parietal, somatosensory, prefrontal, and frontal areas (Jokisch and Jensen, 2007; Medendorp et al., 2007; Haegens et al., 2010; Van Der Werf et al., 2010; Palva et al., 2011), and was found to correlate with individual WM capacity, i.e., the maximal number of items that can be held in WM (Palva et al., 2011). Similarly, source modeling approaches to alpha-band delay activity have reported networks directly involved into WM maintenance (Palva et al., 2011), while other studies showed modulations of alpha-band activity outside the WM network (Jokisch and Jensen, 2007; Medendorp et al., 2007; Haegens et al., 2010; Van Der Werf et al., 2010). Finally, interareal phase synchrony at alpha, beta, and gamma frequencies has been reported to be prominently involved into the maintenance of neural object representations (Palva et al., 2010).

Together, these findings provide important evidence for the functional relationship between neural oscillations in different frequency ranges and the maintenance of information during WM. However, it is currently unclear which cortical networks and frequencies contribute to the maintenance of behaviorally relevant WM information. This question is important because the storage capacity of WM is highly limited (Cowan, 2001), and interindividual differences in WM capacity critically depend on the ability to separate behaviorally relevant from irrelevant information (Vogel et al., 2005).

To examine this question, we recorded MEG data during the delay period of a visuospatial DSM task with distractors that made it possible to identify delay activity that was specific to the maintenance of behaviorally relevant items (see Fig. 1). We employed spectral analysis as well as a beamforming approach to identify the cortical sources of delay activity in different frequency bands. A decoding approach was employed to assess the contribution of delay activity in parietal and prefrontal areas to the maintenance of behaviorally relevant versus irrelevant information.

\section{Materials and Methods}

Participants and visuo-spatial WM task. MEG recordings were collected from 25 (10 females; age range, 18-32 years) adult participants with normal or corrected to normal vision. Written informed consent was obtained before the beginning of the experiment, and all experimental procedures were in agreement with the guidelines of the university's ethics committee. Participants performed a delayed match to sample task (Fig. 1). The sample stimulus consisted of three red discs (load 3), three red and three blue discs (distractors), or six red discs (load 6), which were presented for $0.4 \mathrm{~s}$. This was followed by a maintenance phase of $1.2 \mathrm{~s}$ and the test stimulus ( $0.4 \mathrm{~s})$, a single item whose position was either identical or different from the positions of the items in the sample stimulus. The prestimulus period varied between 3.5 and $1.5 \mathrm{~s}$ to prevent temporal expectation. Participants performed 150 trials per condition, and the order of trials was randomized across conditions. Participants pressed one of two buttons to indicate whether the position of the test item was a match or a nonmatch and were instructed to memorize only the positions of red discs and to ignore blue discs. Hand assignment was counterbalanced across participants, and behavioral responses were recorded 
using a fiber-optic response device (Lumitouch, Photon Control). After responding, participants had the possibility to blink for $1 \mathrm{~s}$ before the next trial started.

MEG data acquisition and preprocessing. MEG signals were recorded continuously using a 275-channel whole-head system (Omega 2005, VSM MedTech) in a synthetic third order axial gradiometer configuration. MEG signals were sampled at $1.2 \mathrm{kHz}$ and bandpass filtered at $0.01-600 \mathrm{~Hz}$. The electrooculogram was recorded to monitor horizontal and vertical eye movements. Before and after each run, the subject's head position relative to the gradiometer array was measured using three fiducial coils (one coil over the nasion and two coils at $1 \mathrm{~cm}$ distance from the left and right tragus). Interindividual deviations of head orientations from the mean remained below $8^{\circ}$ (range for $x$ coordinates, -0.86 to 0.85 $\mathrm{cm}$; range for $y$ coordinates, -1 to $0.88 \mathrm{~cm}$; range for $z$ coordinates: -1.64 to $1.74 \mathrm{~cm})$. MEG signals were preprocessed and analyzed using Matlab 2008b (MathWorks) and the open source Matlab toolbox fieldtrip (http://www.ru.nl/fcdonders/fieldtrip/). Preprocessing included bandpass filtering of the MEG recordings (Butterworth filter fourth order) between 0.1 and $250 \mathrm{~Hz}$ as well as detrending and subtraction of the DC offset. The continuous recording was segmented into data epochs including the baseline ( $1.5 \mathrm{~s})$, the encoding $(0.4 \mathrm{~s})$, and the maintenance $(1.2 \mathrm{~s})$, as well as the recognition ( $0.4 \mathrm{~s})$ phase of the task ( $3.5 \mathrm{~s}$ per trial). Data epochs for match and nonmatch trials were pooled together, and only trials with correct responses were considered for further analyses. An automatic artifact detection and rejection algorithm from the fieldtrip toolbox was used to exclude epochs contaminated by eye blinks, muscle activity, or sensor jump artifacts. The average number of trials per condition across participants included for analysis was 87 of 150 trials $(58 \%)[\mathrm{SD}=23(15 \%)]$.

Structural MRI data acquisition. Structural MRIs were obtained with a 3T Siemens Allegra scanner device (Siemens) using the standard circularly polarized birdcage head-coil and a T1 sequence. Three participants met an exclusion criterion for MRI scans and were not included for source analyses.

Estimation of spectral power and statistical analysis. All spectral quantities were estimated using multitapers as implemented in the Matlabbased open source toolbox Chronux (Mitra and Bokil, 2008) (http:// chronux.org/). The power spectrum of MEG delay activity was estimated using a $1.2 \mathrm{~s}$ window (from 0.4 to $1.6 \mathrm{~s}$ ) with a spectral concentration of $4 \mathrm{~Hz}$ using 4 Slepian data tapers for frequencies below $20 \mathrm{~Hz}$ and with a spectral concentration of $10 \mathrm{~Hz}$ using 10 Slepian data tapers for frequencies above $20 \mathrm{~Hz}$. All spectral quantities of delay activity were normalized by the spectrum of baseline activity and expressed in values of relative change according to

$$
\operatorname{Norm}(f)=(\operatorname{St}(f)-\operatorname{Sb}(f)) / \operatorname{Sb}(f),
$$

where $\operatorname{St}(f)$ corresponds to the spectrum of delay activity and $\operatorname{Sb}(f)$ corresponds to the spectrum of baseline activity. Time-frequency representations of delay activity were calculated using a $500 \mathrm{~ms}$ window that was stepped by $50 \mathrm{~ms}$ between estimates through the trial with the time index aligned to the center of the analysis window. Trials were aligned in time to the onset of the sample stimulus. In analogy to the normalization of the power spectrum, time-frequency representations were normalized by the time-frequency representations of baseline activity according to

$$
\operatorname{Norm}(t, f)=(\operatorname{St}(t, f)-\mathrm{Sb}(\mathrm{tf})) / \mathrm{Sb}(t, f),
$$

where $\mathrm{St}(t, \mathrm{f})$ and $\mathrm{Sb}(t, f)$ correspond to the time-frequency representation of task and baseline activity, respectively. Normalized spectral quantities of delay activity were tested for significant task effects using a cluster-based randomization procedure implemented in the FieldTrip toolbox (Oostenveld et al., 2011).

Task-specific modulations of spectral power in both frequency bands were assessed using a one-way ANOVA for repeated measures. This approach is sensitive to a main effect of the experimental conditions reflecting differences in the means between the load 3, distractor, and load 6 conditions across participants. The alpha level chosen for statistical significance at sensor level was alpha $=0.025$ (corrected) and the alpha level chosen for statistical significance at source level was alpha $=0.001$ (uncorrected). All subsequent steps in the statistical analysis were computed using the MATLAB statistics toolbox. The first $200 \mathrm{~ms}$ of the delay period were not considered for post hoc comparisons of delay activity to exclude encoding related activity captured by half the length of the sliding window. A Bonferroni-correction was applied to post hoc comparisons (twosided dependent $t$ test) to adjust the alpha level for multiple comparisons.

To examine relationships between spectral power, reaction times (RTs), and WM capacity, we selected sensors characterized by significant differences in delay activity across experimental conditions. Power values in the alpha $(10-14 \mathrm{~Hz})$ and gamma $(60-80 \mathrm{~Hz})$ frequency ranges were then correlated with RTs and WM capacity $(\mathrm{K})$.

Beamforming. A frequency domain beamformer (Gross et al., 2001) was used to localize the sources of oscillatory activity in the alpha and gamma frequency bands. The cross-spectral density matrices of each participant were computed for center frequencies at $12 \pm 2 \mathrm{~Hz}$ (alpha) using 3 Slepian data tapers and at $70 \pm 10 \mathrm{~Hz}$ (gamma) using 19 Slepian data tapers based on common filters.

Common filters were computed for trials pooled across conditions on $2 \mathrm{~s}$ time windows, including $1 \mathrm{~s}$ of the baseline period (from -1.6 to -0.6 $\mathrm{s}$ ) and $1 \mathrm{~s}$ of the maintenance phase (from 0.6 to $1.6 \mathrm{~s}$ ). The beamformer was then computed for baseline and delay time segments of $1 \mathrm{~s}$ for each condition separately with a regularization parameter of lambda $=1 \%$. Poststimulus activation effects were normalized with baseline activity according to

$$
\operatorname{Norm}(x, y, z)=(\operatorname{Bt}(x, y, z)-\mathrm{Bb}(x, y, z)) / \mathrm{Bb}(x, y, z),
$$

where $\operatorname{Bt}(x, y, z)$ corresponds to the beamformer of the delay phase and $\mathrm{Bb}(x, y, z)$ corresponds to the beamformer of baseline activity. Normalized source activity was tested for significant task effects using the clusterbased randomization procedure described earlier.

Computation of source grids. The forward solution for each participant was estimated from individual head models using a common dipole grid in MNI space that was warped onto the anatomy of each participant and a realistic single-shell volume conductor model (Nolte, 2003). Cortical segmentation and surface reconstruction were performed using SPM2 (http://www.fil.ion.ucl.ac.uk/spm) as well as spatial alignment of structural MRIs and functional maps. We computed between-subjects cluster-based randomization test statistics (Maris and Oostenveld, 2007) to measure task effects across conditions for each grid point and each frequency band. Only the peak voxels were selected for the subsequent reconstruction of virtual channels.

Computation of virtual channels. For each frequency band, the sensorlevel data were bandpass filtered using a fourth-order, zero-phase, forward-reverse, digital Butterworth filter, and source-level time courses of these band-limited signals were reconstructed using the real-valued frequency-specific beamformer coefficients. The dominant dipole direction of the time courses was then extracted using eigenvalue decomposition, and spectral power for each frequency band was computed from band-limited time-frequency representation using multitaper.

Single trial decoding of gamma-band source activity. Sixty to eighty hertz single trial source activity was decoded from the integral of the power time course in this frequency band during the memory period (from 0.6 to $1.6 \mathrm{~s}$ ) using linear discriminant analysis (Duda et al., 2001). The discriminant function was trained on a random selection of trials $(90 \%)$, while the remaining trials (10\%) were used to assess the classification hit rate. This procedure was repeated 1000 times, and the probability of a correct classification was expressed as the average classification hit rate across iterations. To assess the statistical significance of the classification results, we computed D-prime values for the decoding of load 3 and load 6 trials for each participant. We computed $d^{\prime}$ as previously proposed (Stanislaw and Todorov, 1999):

$$
d^{\prime}=z(\mathrm{P}(\mathrm{Y} \mid s))-z(\mathrm{P}(\mathrm{Y} \mid n))
$$

with $z(\mathrm{P}(\mathrm{Y} \mid s))$ being the $z$-score of the hit rate, and $z(\mathrm{P}(\mathrm{Y} \mid n))$ being the $z$-score of the false alarm rate. We defined the hit rate as the proportion of correctly classified trials, and the false alarm rate as the proportion of 
Table 1. Means and standard deviations for behavioral performance

\begin{tabular}{lccc}
\hline & \multicolumn{4}{l}{ Experimental conditions } \\
\cline { 2 - 4 } Behavioral measure & Load 3 & Distractor & Load 6 \\
\hline Hit rate (\%) & $95.7(5.4)$ & $95.4(4.5)$ & $86.1(11.2)$ \\
RTs (ms) & $653.9(154.4)$ & $663.6(151.3)$ & $710(163.5)$ \\
\hline
\end{tabular}

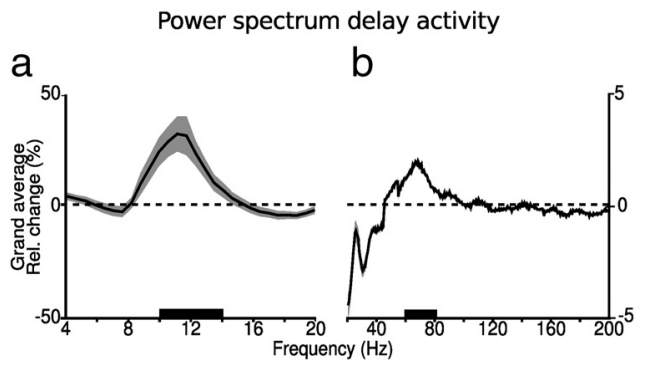

Figure 2. MEG power spectrum of delay activity. $\boldsymbol{a}$, Power spectrum for frequencies from 4 to $20 \mathrm{~Hz} . \boldsymbol{b}$, Power spectrum for frequencies from 20 to $200 \mathrm{~Hz}$. $x$-axis represents frequency and $y$-axis represents power. Spectral power is expressed in percent change relative to baseline activity. The solid horizontal black lines mark the frequency bands considered for analysis. The shaded area around the curve corresponds to SEM.

trials that were falsely classified. Thus for load 3 the hit rate was computed as the proportion of correctly decoded load 3 trials, and the false alarm rate as the proportion of load 6 trials that were falsely classified as load 3 and vice versa. This approach was applied to the results of the single trial decoding for gamma-band delay activity in the intraparietal lobule (IPL) and Brodmann area 9 (BA9) for each participant.

\section{Results}

\section{Behavioral performances}

The hit rate and reaction times were analyzed for the three different conditions (Table 1). There was a significant difference in the hit rates $\left(\chi_{(2,777)}^{2}=21.9, p<0.0001\right.$, Kruskal-Wallis one-way ANOVA). Post hoc comparisons revealed a decrease in the hit rate between load 3 and load $6(z=4.19, p<0.0001$, post hoc MannWhitney $U$ test; corrected), as well as between load 6 and the distractor condition $(z=3.83, p<0.001$, post hoc Mann-Whitney $U$ test; corrected). No differences were found for hit rates in the load 3 and distractor conditions $(z=0.44, p>0.05$, post hoc Mann-Whitney $U$ test; corrected). Furthermore, no differences were found for reaction times $\left(F_{(2,77)}=0.9, p>0.05\right.$, one-way ANOVA).

\section{Spectral power at sensor level}

Delay activity revealed a peak in the alpha $(10-14 \mathrm{~Hz})$ and gamma $(60-80 \mathrm{~Hz})$ frequency ranges. In both frequency bands, activity was significantly elevated above baseline ( $p<0.001$; corrected; $t$ test; Fig. $2 a, b)$ and modulated across task conditions $(p<0.025$; corrected; ANOVA). Delay activity between 0.6 and $1.6 \mathrm{~s}$ showed a significant increase of gamma-band power during load 6 as compared with both load 3 and the distractor condition $(p<0.001$; corrected; post hoc $t$ test; Fig. $3 a, b)$, while there was no difference between the distractor condition and load 3 ( $p>0.05$; post hoc $t$ test).

The increase in gamma-band power between load 3 and load 6 was correlated with WM capacity $(r=0.48, p<0.05)$, i.e., the maximal number of items each individual could hold in WM (Rouder et al., 2011). In addition, there was a significant relationship between the increase in gamma-band power between load 3 and load 6 and the difference in reaction times between both conditions $(r=-0.46, p<0.05)$. A similar correlation was found for the increase in gamma-band power between load 6 and the distractor condition and WM capacity (Fig. 4a,b; Table 2). Gamma-band delay activity in each condition alone, however, did not correlate with reaction times (load 3, $r=0.22 p>0.05$; distractor, $r=0.3, p>0.05$; load6, $r=-0.03 ; p>0.05)$.

Alpha-band delay activity was characterized by a different relationship with WM load and reaction times (Fig. 4a, Table 2). While spectral power was increased in load 6 and the distractor condition relative to load 3 ( $p<0.01$; corrected; post hoc $t$ test; Fig. $5 b$ ), there was no difference between load 6 and the distractor condition ( $p>0.05$; post hoc $t$ test). Alpha-band power in the distractor $(r=0.46, p<0.05)$ and load $6(r=0.47, p<0.05)$ conditions showed a positive correlation with reaction times (Fig. $4 c, d)$, but there was no relationship between the increase in alpha-band delay activity between load 3 and load 6 as well as between the distractor condition and load 6 and individual WM capacity (Table 2).

We did not observe significant changes relative to baseline in the theta $(4-7 \mathrm{~Hz})$ and beta bands $(15-30 \mathrm{~Hz}$; Fig. 2a,b), except for a transient increase of beta oscillations $(20-28 \mathrm{~Hz})$ from 0.75 to $1.2 \mathrm{~s}$ (Fig. $3 a$ ). This beta activity was positively correlated with alpha-band oscillations across conditions and participants (average correlation coefficient $r=0.55, \mathrm{SD}=0.3$ ). Thus, $20-28 \mathrm{~Hz}$ delay activity likely reflects harmonics of oscillations in the 10-14 $\mathrm{Hz}$ range.

\section{Spectral power at source level}

Task-related differences in gamma-band power $(p<0.001$; uncorrected; ANOVA) were localized to left BA9 (MNI coordinates: $-2,52,40)$ and right IPL (MNI coordinates: 30, -48, 32). Gamma-band power in BA9 was increased during the load 6 condition as compared to the distractor and load 3 conditions $(p<0.001$; corrected; post hoc $t$ test; Fig. $6 a, b)$. However, there was no difference between load 3 and the distractor condition ( $p>0.05$; post hoc $t$ test). In the IPL, gamma-band power was enhanced in the load 6 and distractor conditions relative to load 3 $(p<0.001$; corrected; post hoc $t$ test; Fig. $6 c, d)$ but reached similar amplitudes in the load 6 and the distractor condition $(p>0.05$; post hoc $t$ test).

Task effects $(p<0.001$; uncorrected; ANOVA) in the alpha band were localized to BA6 (MNI coordinates: 42, 10, 58; Fig. $6 e)$, which contributes to premotor cortex. Alpha power in BA6 increased in the load 6 and distractor conditions relative to load 3 ( $p<0.001$; corrected; post hoc $t$ test). As for gammaband power in the IPL, alpha-band activity in BA6 did not differ between load 6 and the distractor condition $(p>0.05$; post hoc $t$ test; Fig. 6e).

\section{Predicting WM load from single trial $60-80 \mathrm{~Hz}$ source activity}

To identify the cortical area whose activation predicts the number of items maintained in WM, linear discriminant analysis was applied to $60-80 \mathrm{~Hz}$ activity during behaviorally successful trials in BA9 and IPL. We first decoded gamma activity from load 3 and load 6 trials and then applied the same classifier to gamma-band activity during distractor trials to estimate the probability of distractor trials being classified as load 3 and load 6 across trials for each participant. Assuming that $60-80 \mathrm{~Hz}$ oscillations reflect the maintenance of behaviorally relevant items in WM, we predicted that gamma-band activity during behaviorally successful trials 


\section{0-120 Hz activity at sensor level}

a

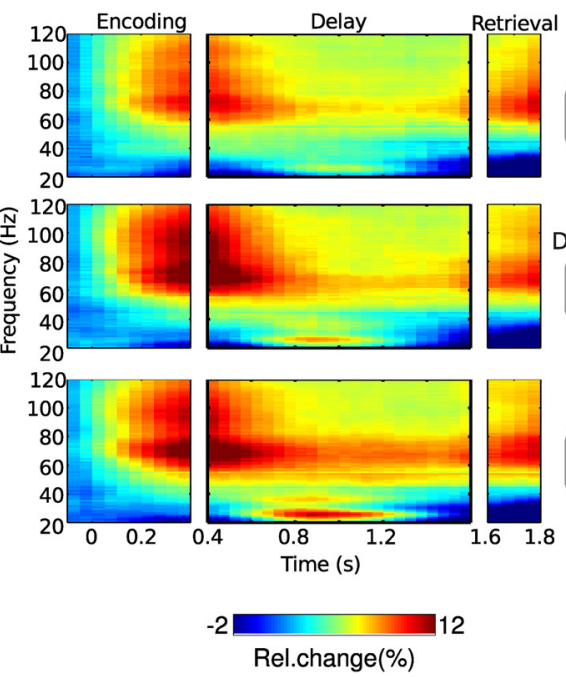

b

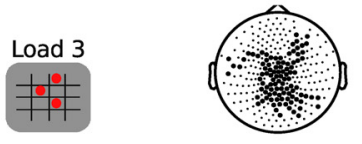

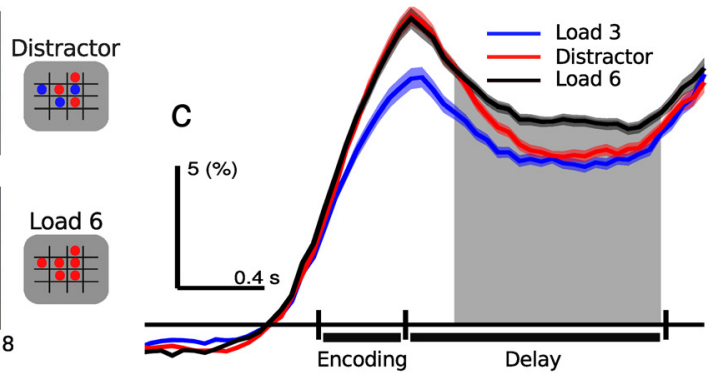

Figure 3. MEG spectrograms for $20-120 \mathrm{~Hz}$ activity. $\boldsymbol{a}$, Two-dimensional plots of the spectrograms for each condition. Note the transient increase of $10-14 \mathrm{~Hz}$ harmonic activity in the $20-28$ $\mathrm{Hz}$ range. $x$-axis represents time and $y$-axis represents frequency. Spectral power is expressed in percent change relative (Rel. change) to baseline activity. $\boldsymbol{b}$, Gamma-band activity was significantly modulated by task conditions in 100 (38\%) sensors ( $p<0.025$; corrected; ANOVA). $c, 60-80 \mathrm{~Hz}$ activity averaged across trials. The shaded area around the traces corresponds to the SEM. The light gray region marks the temporal interval of significant differences between conditions $(p<0.001$; corrected; post hoc $t$ test).

\section{Correlations between spectral power and behavioral performances}
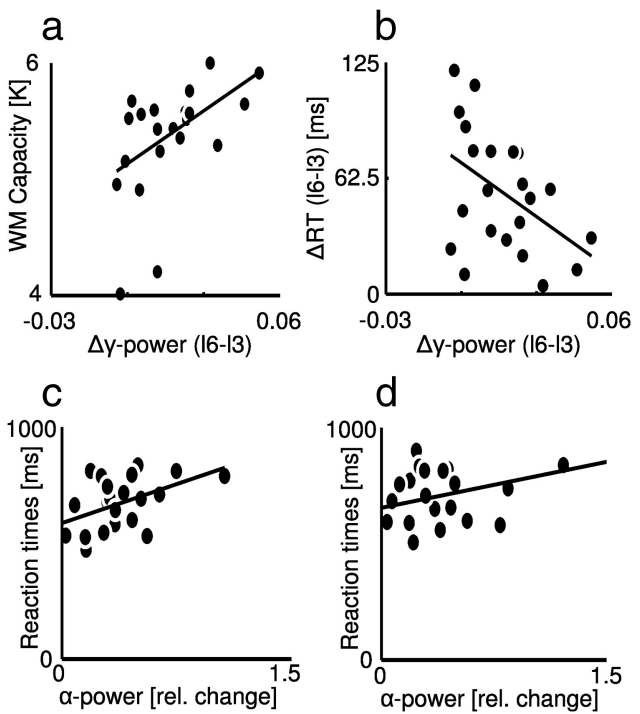

Figure 4. Relationship between spectral power and behavioral performances. $\boldsymbol{a}$, Linear regression between the increase of gamma band $(60-80 \mathrm{~Hz})$ delay activity from load 6 relative to load 3 and WM capacity. $\boldsymbol{b}$, Linear regression between the increase of $60-80 \mathrm{~Hz}$ delay activity from load 6 relative to load 3 and the increase in reaction times from load 6 to load 3. c, Linear regression between alpha-band $(10-14 \mathrm{~Hz})$ delay activity and reaction times in the distractor condition. $\boldsymbol{D}$, Linear regression between 10 and $14 \mathrm{~Hz}$ delay activity and reaction times in the load 6 condition. rel. change, Relative change.

with distractors should have a higher probability to be classified as load 3 than as load 6 .

The average probability of a correct assignment of load 3 trials was 59\% (SD across participants, 10\%) in BA 9 and 56\% (SD across participants, 10\%) in the IPL. Load 6 trials were correctly decoded with an average probability of 55\% (SD across partici-
Table 2. Correlations between spectral power at sensor level and behavioral performances

\begin{tabular}{lcc}
\hline Difference in delay activity & WM capacity $(\mathrm{K})$ & Reaction time difference $(\mathrm{ms})$ \\
\hline Load 6 - Load 3 & & \\
$10-14 \mathrm{~Hz}$ & -0.39 & 0.27 \\
$60-80 \mathrm{~Hz}$ & $0.49^{*}$ & $-0.46^{*}$ \\
Load 6 - Distractor & & \\
$10-14 \mathrm{~Hz}$ & -0.26 & 0.18 \\
$60-80 \mathrm{~Hz}$ & 0.3 & -0.31
\end{tabular}

WM-capacity indexes individual WM capacity for load 6 . Reaction time differences reflect the individual increase in RTs between load 6 and load 3 as well as between load 6 and the distractor condition. ${ }^{*} p<0.05$.

pants, $8 \%$ ) in BA 9 and 57\% (SD across participants, 9\%) in the IPL (Fig. 7a). The decoding performance reported in the present study corresponds to performance levels reported by classification approaches in fMRI data (Knops et al., 2009). Decoding performance for gamma-band source activity in the IPL was not significantly different from zero across participants for load 3 $\left(t_{(21)}=1.6, p>0.05\right)$. However, decoding of delay activity for load 6 trials in the IPL was significant $\left(t_{(21)}=3.35, p<0.01\right)$. In BA9, the distribution of $d^{\prime}$ values was significantly different from zero across participants in both conditions (load 3: $t_{(21)}=3.29$, $p<0.01$; load 6: $\left.t_{(21)}=3.1, p<0.01\right)$.

To test the hypothesis that $60-80 \mathrm{~Hz}$ oscillations are correlated with the maintenance of behaviorally relevant WM items, we predicted class membership (load 3 vs load 6) for distractor trials using the same discriminant function as for the classification of load 3 and load 6 trials. We found that gamma-band activity in BA 9 predicted the amount of behaviorally relevant information maintained in WM with 17 of 22 (77\%) participants showing a probability of distractor trials being classified as load 3 greater than chance $(p<0.01$; corrected; two-sided binomial test). In IPL, distractor trials were classified as load 3 trials in only $10(45 \%)$ of 22 participants $(p=>0.05$; two-sided binomial test). The mean classification probability for distractor trials in the IPL to be classified as load 3 was $49 \%$ (SD, 15\%), whereas in BA9 the mean probability of distractor trials to be classified as 
10-14 $\mathrm{Hz}$ activity at sensor level
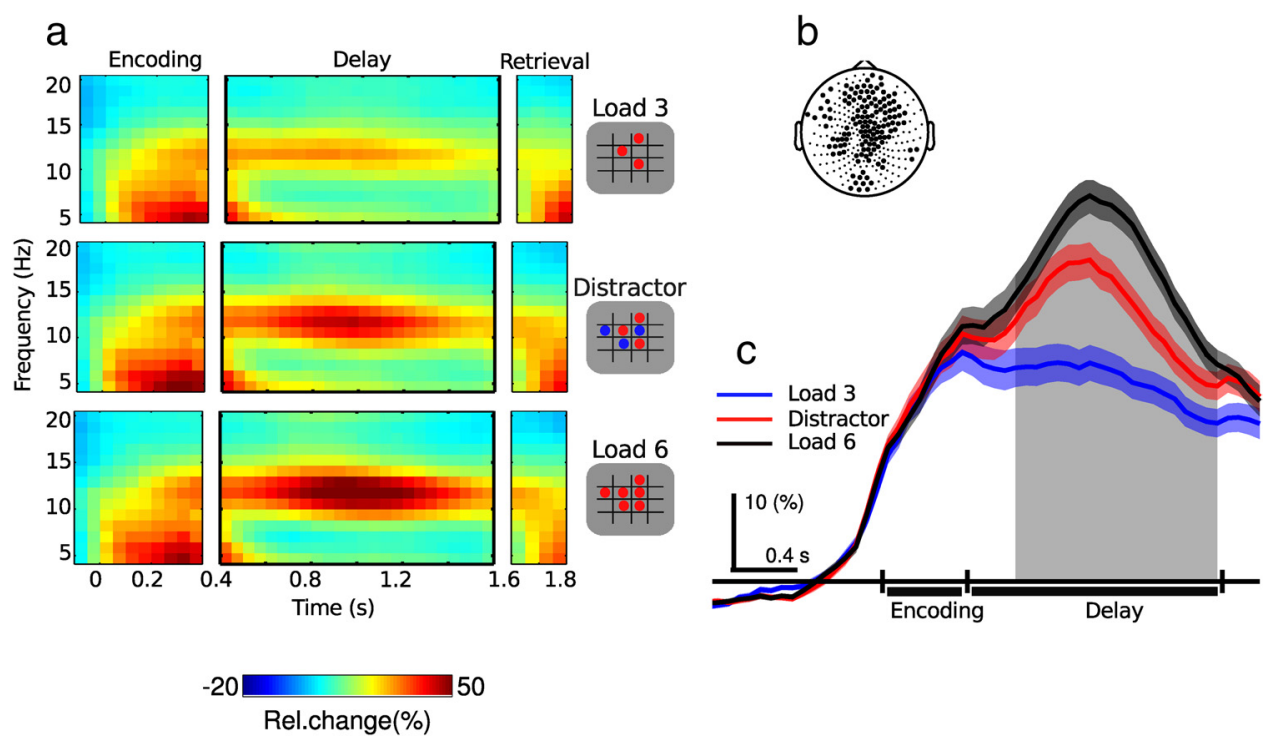

Figure 5. MEG spectrograms for $6-20 \mathrm{~Hz}$ activity (same convention as in Fig. 2). $\boldsymbol{a}$, Two-dimensional plots of the spectrograms for each condition. $x$-axis represents time and $y$-axis represents frequency. Spectral power is expressed in percent change relative to baseline activity. $\boldsymbol{b}$, Alpha-band power was significantly modulated across task conditions in $137(58 \%)$ sensors $(p<0.025$; corrected; ANOVA). $c$, Ten to fourteen hertz activity averaged across trials ( $p<0.01$; corrected; post hoc $t$ test). Rel. change, Relative change.

load 3 was 59\% (SD, 19\%). Furthermore, the probability of distractor trials to be classified as load 3 was significantly higher in BA 9 as compared to the IPL ( $p<0.01$, two-sided $t$ test). These results suggest that the number of behaviorally relevant items maintained in WM can be decoded from 60 to $80 \mathrm{~Hz}$ source power in BA 9 (Fig. 7b).

\section{Discussion}

In the present study we investigated the relationship between oscillatory activity in the $4-200 \mathrm{~Hz}$ frequency-range and the maintenance of behaviorally relevant WM representations. Our findings show that gamma-band $(60-80 \mathrm{~Hz})$ delay activity in left prefrontal cortex (PFC) codes for the number of relevant items maintained in WM, because neither $60-80 \mathrm{~Hz}$ activity in parietal cortex nor alpha $(10-14 \mathrm{~Hz})$ delay activity in BA 6 was characterized by a consistent relationship between oscillation amplitude and the number of target items. Thus, our study suggests that gamma oscillations in PFC are critically implicated in the maintenance of relevant WM information.

\section{WM-delay activity of alpha and gamma-band oscillations at sensor level}

In line with previous studies (Tallon-Baudry et al., 1998; Pesaran et al., 2002; Howard et al., 2003; Jokisch and Jensen, 2007; Meltzer et al., 2008; Haegens et al., 2010; Van Der Werf et al., 2010), we report an increase of oscillatory activity in the gamma frequency band during the retention phase. Furthermore, 60-80 Hz delay activity was observed to increase with WM load, which is consistent with data obtained from intracortical recordings in humans (Howard et al., 2003).

Interestingly, when only three of six presented items were relevant, the analysis of gamma-band activity revealed a correspondence between the oscillation amplitude and the number of target items. This relationship was not found for spectral power in the alpha band because a similar amplitude increase occurred between the distractor condition and load 6, suggesting that only $60-80 \mathrm{~Hz}$ delay activity is sensitive to the number of relevant
WM items. Furthermore, correlations with RTs and WMcapacity suggest that gamma-band activity had a facilitatory effect on WM performance.

This relationship was not found for alpha-band activity. Consistent with previous studies (Jensen et al., 2002; Meltzer et al., 2008; Sauseng et al., 2009; Palva et al., 2011), alpha-band oscillations were modulated by WM load because $10-14 \mathrm{~Hz}$ activity increased between load 3 and load 6 but correlated negatively with RTs. Together with previous evidence that has interpreted alpha-band activity over motor areas in human EEG data as the inhibition of motor areas (Pfurtscheller et al., 1997) and studies showing that alpha-band delay activity increases over task-irrelevant brain regions (Pfurtscheller et al., 1997; Worden et al., 2000; Jokisch and Jensen, 2007; Medendorp et al., 2007; Sauseng et al., 2009; Haegens et al., 2010), our data suggest that alpha-band oscillations are not involved in the active maintenance of WM representations. Instead, a more likely function of increased $10-14$ activity could be the inhibition of cortical areas involved into the preparation or execution of motor actions. This interpretation is consistent with reports of focally increased $11-13 \mathrm{~Hz}$ activity during the inhibition of motor memory traces (Hummel et al., 2002) and the association between increased alpha-band power and slower behavioral responses in the current study.

\section{Alpha- and gamma-band WM networks}

Further support for the distinct contributions of alpha- and gamma-band oscillations toward the maintenance of relevant WM representations was obtained through the identification of the underlying cortical sources. Previous MEG studies have reported a WM load-specific strengthening of oscillatory delay activity in prefrontal and parietal areas that correlated with individual WM capacity (Palva et al., 2011), suggesting that activity in these brain areas is implicated in the maintenance of WM representations. In agreement with these findings, we report WM-related changes of gamma-band source activity in the left BA9 and the right IPL. 


\section{Gamma-band $(70+/-10 \mathrm{~Hz})$ and alpha-band $(12+/-2 \mathrm{~Hz})$ source power}
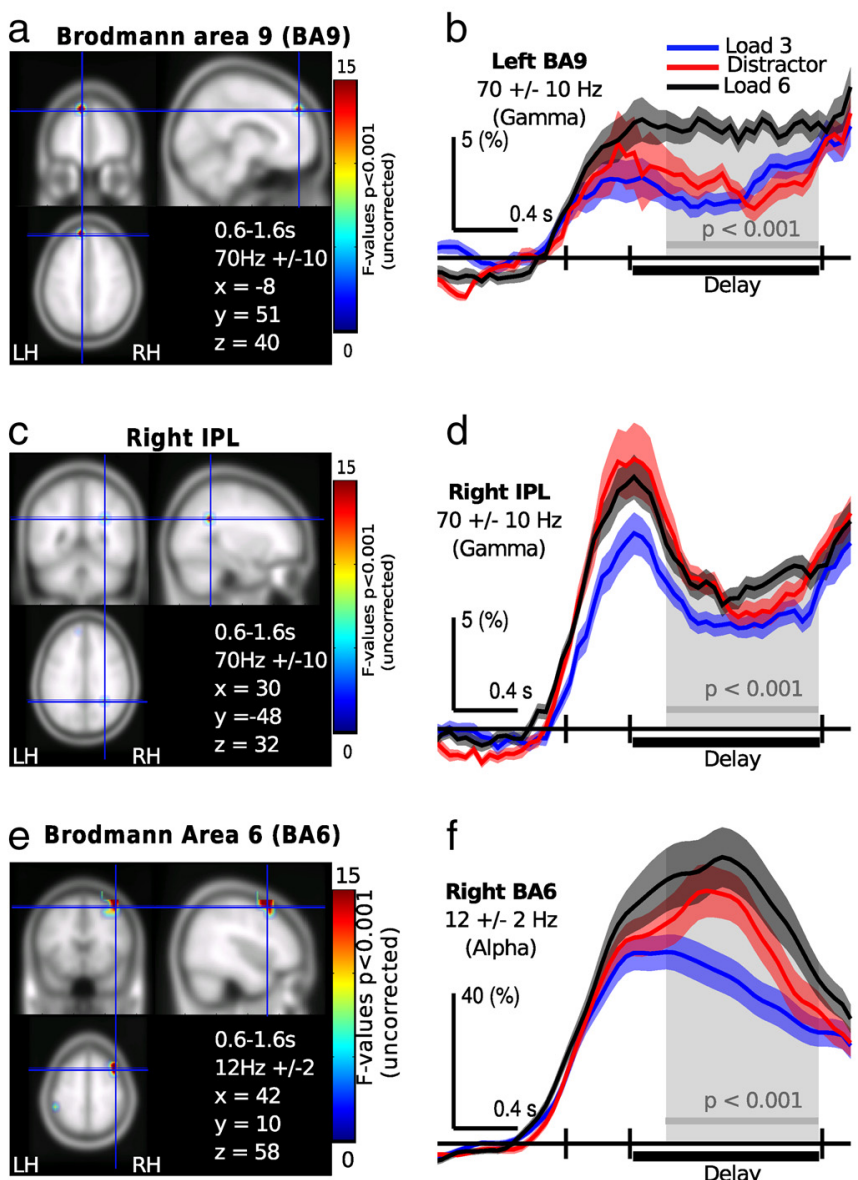

Figure 6. Delay activity at cortical source level. $\boldsymbol{a}, \boldsymbol{b}$, Images of the differences in $60-80 \mathrm{~Hz}$ activity (0.6 to $1.6 \mathrm{~s}$ ) across task conditions during the delay period for the left BA $9(\boldsymbol{a})$ and the intraparietal lobule $(\boldsymbol{b})$. Effects are separately shown for the two brain regions and are displayed on axial, sagittal, and coronal sectional views of the MNI template brain. The locations of BA 9 and IPL are marked for orientation. All functional maps display dependent $F$ values thresholded at $p<0.001$ (uncorrected). $\boldsymbol{c}, \boldsymbol{d}$, Time course of $60-80 \mathrm{~Hz}$ activity for peak voxels averaged across trials in BA 9 ( $\boldsymbol{c}$ ) and IPL (d). The light gray region corresponds to the temporal interval of significant differences between conditions ( $p<0.001$; corrected; post hoc $t$ test). $c$, In BA9 there was a significant increase of $60-80 \mathrm{~Hz}$ activity from 0.6 to $1.6 \mathrm{~s}$ during load 6 as compared to the load 3 and distractor conditions, while activity during load 3 and the distractor was similar. $\boldsymbol{d}$, In contrast, in the IPL $60-80 \mathrm{~Hz}$ delay activity was increased during load 6 and the distractor as compared to load 3 and did not differ between the distractor and load 6 condition. $\boldsymbol{e}$, Statistical map of the differences in $10-14 \mathrm{~Hz}$ activity ( 0.6 to $1.6 \mathrm{~s}$ ) across task conditions during the delay period for Brodmann area 6 .f, Time course of $10-14 \mathrm{~Hz}$ source activity averaged across trials for BA 6 . Source activity in the $10-14 \mathrm{~Hz}$ range was significantly elevated during the delay ( 0.6 to $1.6 \mathrm{~s}$ ) in the distractor and load 6 conditions as compared to load 3 and remained similar in the distractor and load 6 conditions.

In both cortical regions we observed an increase of gammaband delay activity with WM load. However, the analysis of source activity revealed that the correspondence between oscillation amplitude and the number of target items was specific to activity in BA9. This was confirmed by a decoding approach applied to single-trial delay activity at source level which showed that $60-80 \mathrm{~Hz}$ activity in BA9 covaried with the number of relevant items, which was not the case in the IPL.

Our results thus suggest a specific contribution of gammaband activity in left BA9 toward the maintenance of relevant items in visuospatial WM. This conclusion is consistent with electrophysiological recordings in nonhuman primates (Rainer et al., 1998) and lesion studies in humans (du Boisgueheneuc et al., 2006) and nonhuman primates (Funahashi et al., 1993), as well as the specific effect of disrupting WM maintenance through transcranial magnetic stimulation in PFC (Feredoes et al., 2011), which highlight the importance of left PFC for the maintenance of behaviorally relevant WM representations.

In the alpha band, task effects were localized to right BA6, a region comprising the premotor cortex and the supplementary motor area (SMA), which has been shown to be critically involved in the planning and inhibition of motor plans (Sumner et al., 2007). These results further support the interpretation of $10-14 \mathrm{~Hz}$ delay activity as a mechanism to protect memory traces from interfering preparatory motor activity.

\section{Relationship to previous neurophysiological WM theories and empirical findings}

Previous work suggests that successful WM maintenance involves a distributed network of cortical areas (Miller and Desimone, 1994; Palva et al., 2010, 2011). In line with these findings, we report delay activity at alpha- and gamma-band frequencies in parietal and frontal regions. However, our data suggests that only gamma-band activity in BA 9 contributes toward the maintenance of behaviorally relevant WM items.

WM delay activity at gamma and alpha-band frequencies in distinct nodes of the WM-network in our study can be integrated with recent data from invasive and noninvasive electrophysiological recordings. Thus, gamma-band activity in parietal cortices could be related to topographic maps that code for the spatial memories of saccades (Blatt et al., 1990; Ben Hamed et al., 2001). This is suggested by the fact that gamma-band activity in IPL was modulated by the number of stimuli presented in the memory array but not by the number of relevant items, which suggests that the modulation of $60-80 \mathrm{~Hz}$ activity reflects a scanning and maintenance of the spatial position of possible WM locations.

Accordingly, gamma-band activity in IPL may reflect the activity of memory fields (Funahashi et al., 1989) that have been suggested to synchronize at gamma frequencies to strengthen their input on downstream targets in the oculomotor network (Van Der Werf et al., 2010). Based on this hypothesis, we propose that the coupling between PFC-IPL through coherent oscillations (Fries, 2005) may serve as a link for the readout of behaviorally relevant stimuli positions. This interpretation is consistent with memory fields in the PFC which have been reported to be critically implicated in the control of memory guided oculomotor responses during WM (Funahashi et al., 1993) and with our findings that fluctuations of 
gamma-band activity in PFC allowed the decoding of the number behaviorally relevant items.

The interpretation of gamma-band delay activity as the maintenance and readout of spatial maps coding for WM locations is further supported by the localization of alpha-band delay activity in BA6. Transcranial magnetic stimulation of SMA has been observed to impair the ability to reproduce memorized gaze positions (Müri et al., 1995), and neurons in pre-SMA have been implicated in the organization of oculomotor sequences (Isoda and Tanji, 2004). From this perspective, alpha-band oscillations in SMA in our study could be involved in the inhibition of oculomotor movements, as these could interfere with WM locations maintained in parietal cortex.

The distinct roles of alpha- and gamma-band oscillations in the present study suggest that these findings may be specific to visuospatial WM and may not apply to other WM paradigms. This could be one explanation for the absence of significant task effects in the theta band in our study that has been prominently implicated in neurophysiological WM theories (Lisman, 2010). Previous studies that have reported a relationship between WM load and the modulation of theta-band oscillations have investigated WM-delay activity during tasks that typically require the maintenance of sequential WM representations, i.e., Sternberg tasks (Jensen and Tesche, 2002).

\section{Methodological limitations of source localization}

The pattern of lateralization of both alpha- and gamma-band activity can be considered consistent with previous studies that have emphasized the role of the left PFC for the maintenance of relevant items in WM (Rainer et al., 1998; du Boisgueheneuc et al., 2006). In addition, right IPL activity has been shown to be critical for visuospatial WM (Yamanaka et al., 2010), while the lateralization of activation foci to the right hemisphere occurs with increasing executive demands that could account for increased alpha-band delay activity in BA 6 (Wager and Smith, 2003).

We would like to note, however, that we cannot completely exclude the possibility that the pattern of source localizations may have been affected by the constrains of the spatial beamformer employed in the current study. Although the estimation of neural source signals using inverse modeling reduces the cross talk between sensors at the scalp surface, the output of spatial beamformers can suffer from signal cancellation and cross-talk effects that can affect the identification of sources (Gross et al., 2001).

\section{Conclusion}

The aim of the current study was to identify the frequencies and cortical regions that contribute to the maintenance of behaviorally relevant WM representations. The present findings provide novel evidence for a frequency and anatomically

\section{Working memory decode}

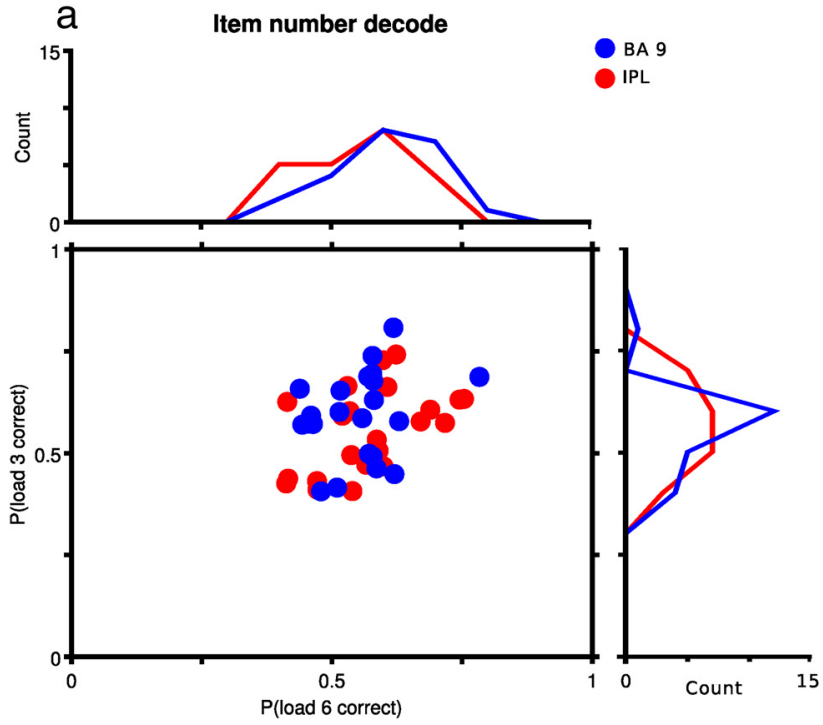

b

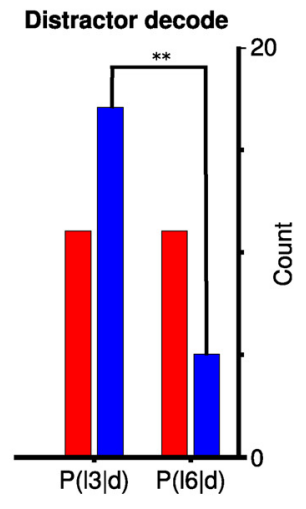

Figure 7. Single trial decoding of $60-80 \mathrm{~Hz}$ delay activity. $\boldsymbol{a}$, Each dot represents classification performance in BA 9 (blue) and for each participant. Horizontal axis is the probability that a trial corresponding to load 6 is decoded correctly. Vertical axis $6(p=$ n.s.; two-sided binomial test).

specific relationship between 60 and $80 \mathrm{~Hz}$ activity in the left BA9 and the maintenance of relevant WM representations. In addition, the current study provides evidence for spectral changes at alpha- and gamma-band frequencies in parietal cortex and premotor cortex. However, delay activity in these areas was not found to be implicated in the maintenance of relevant WM items.

Our findings also highlight that MEG source data can be used to predict the amount of behaviorally relevant information maintained in WM from single-trial fluctuations of gamma-band oscillations in PFC. Studies with fMRI have shown that fluctuations in the BOLD signal can decode the content of WM from delay activity in early visual areas (Harrison and Tong, 2009). The present results suggest that MEG source data can be used as well to predict the content of WM, suggesting that noninvasively recorded brain oscillations allow functional links between oscillatory activity and cognitive processes.

In future studies, it will be interesting to investigate coherence between oscillations in the IPL and BA9 to test whether the readout of relevant information is mechanistically implemented through gamma-band synchronization between memory fields in parietal and prefrontal areas. In addition, decoding approaches applied to eye position data in combination with spectral measures of delay activity in parietal cortex and prefrontal cortex may help to further clarify the mechanistic function of gamma-band activity in the IPL and BA9.

Finally, we believe that the current findings are also important for future research that investigates the pathophysiology of neuropsychiatric disorders such as schizophrenia. Aberrant PFC activity has been linked to recall deficits in working memory (Goldberg et al., 1989), a core deficiency in schizophrenia. In addition, there is evidence that gamma-band activity is 
impaired in patients with schizophrenia (Uhlhaas and Singer, 2010), suggesting a possible contribution of dysfunctional gamma-band oscillations in PFC toward WM impairments in the disorder.

\section{References}

Axmacher N, Henseler MM, Jensen O, Weinreich I, Elger CE, Fell J (2010) Cross-frequency coupling supports multi-item working memory in the human hippocampus. Proc Natl Acad Sci U S A 107:3228-3233.

Baddeley A (2003) Working memory: looking back and looking forward. Nat Rev Neurosci 4:829-839.

Ben Hamed S, Duhamel JR, Bremmer F, Graf W (2001) Representation of the visual field in the lateral intraparietal area of macaque monkeys: a quantitative receptive field analysis. Exp Brain Res 140:127-144.

Blatt GJ, Andersen RA, Stoner GR (1990) Visual receptive field organization and cortico-cortical connections of the lateral intraparietal area (area LIP) in the macaque. J Comp Neurol 299:421-445.

Busch NA, Herrmann CS (2003) Object-load and feature-load modulate EEG in a short-term memory task. Neuroreport 14:1721-1724.

Buzsáki G, Draguhn A (2004) Neuronal oscillations in cortical networks. Science 304:1926-1929.

Cowan N (2001) The magical number 4 in short-term memory: a reconsideration of mental storage capacity. Behav Brain Sci 24:87-114.

du Boisgueheneuc F, Levy R, Volle E, Seassau M, Duffau H, Kinkingnehun S, Samson Y, Zhang S, Dubois B (2006) Functions of the left superior frontal gyrus in humans: a lesion study. Brain 129:3315-3328.

Duda RO, Hart PE, Stork DG (2001) Pattern classification, Ed 2. New York: Wiley.

Feredoes E, Heinen K, Weiskopf N, Ruff C, Driver J (2011) Causal evidence for frontal involvement in memory target maintenance by posterior brain areas during distracter interference of visual working memory. Proc Natl Acad Sci U S A 108:17510-17515.

Fries P (2005) A mechanism for cognitive dynamics: neuronal communication through neuronal coherence. Trends Cogn Sci 9:474-480.

Fuentemilla L, Penny WD, Cashdollar N, Bunzeck N, Düzel E (2010) Thetacoupled periodic replay in working memory. Curr Biol 20:606-612.

Funahashi S, Bruce CJ, Goldman-Rakic PS (1989) Mnemonic coding of visual space in the monkey's dorsolateral prefrontal cortex. J Neurophysiol 61:331-349.

Funahashi S, Bruce CJ, Goldman-Rakic PS (1993) Dorsolateral prefrontal lesions and oculomotor delayed-response performance: evidence for mnemonic "scotomas." J Neurosci 13:1479-1497.

Goldberg TE, Weinberger DR, Pliskin NH, Berman KF, Podd MH (1989) Recall memory deficit in schizophrenia. A possible manifestation of prefrontal dysfunction. Schizophr Res 2:251-257.

Gross J, Kujala J, Hamalainen M, Timmermann L, Schnitzler A, Salmelin R (2001) Dynamic imaging of coherent sources: Studying neural interactions in the human brain. Proc Natl Acad Sci U S A 98:694-699.

Haegens S, Osipova D, Oostenveld R, Jensen O (2010) Somatosensory working memory performance in humans depends on both engagement and disengagement of regions in a distributed network. Human Brain Mapp 31:26-35.

Harrison SA, Tong F (2009) Decoding reveals the contents of visual working memory in early visual areas. Nature 458:632-635.

Hebb DO (1949) The organization of behavior: a neuropsychological theory. New York: Wiley.

Herrmann CS, Senkowski D, Röttger S (2004) Phase-locking and amplitude modulations of EEG alpha: Two measures reflect different cognitive processes in a working memory task. Exp Psychol 51:311-318.

Howard MW, Rizzuto DS, Caplan JB, Madsen JR, Lisman J, AschenbrennerScheibe R, Schulze-Bonhage A, Kahana MJ (2003) Gamma oscillations correlate with working memory load in humans. Cereb Cortex 13:1369-1374.

Hummel F, Andres F, Altenmüller E, Dichgans J, Gerloff C (2002) Inhibitory control of acquired motor programmes in the human brain. Brain 125:404-420.

Isoda M, Tanji J (2004) Participation of the primate presupplementary motor area in sequencing multiple saccades. J Neurophysiol 92:653-659.

Jensen O, Tesche CD (2002) Frontal theta activity in humans increases with memory load in a working memory task. Eur J Neurosci 15:1395-1399.

Jensen O, Gelfand J, Kounios J, Lisman JE (2002) Oscillations in the alpha band $(9-12 \mathrm{~Hz})$ increase with memory load during retention in a shortterm memory task. Cereb Cortex 12:877-882.

Jensen O, Kaiser J, Lachaux JP (2007) Human gamma-frequency oscillations associated with attention and memory. Trends Neurosci 30: 317-324.

Jokisch D, Jensen O (2007) Modulation of gamma and alpha activity during a working memory task engaging the dorsal or ventral stream. J Neurosci 27:3244-3251.

Knops A, Thirion B, Hubbard EM, Michel V, Dehaene S (2009) Recruitment of an area involved in eye movements during mental arithmetic. Science 324:1583-1585.

Leiberg S, Lutzenberger W, Kaiser J (2006) Effects of memory load on cortical oscillatory activity during auditory pattern working memory. Brain Res 1120:131-140.

Lisman J (2010) Working memory: the importance of theta and gamma oscillations. Curr Biol 20:R490-R492.

Lisman JE, Idiart MA (1995) Storage of $7 \pm 2$ short-term memories in oscillatory subcycles. Science 267:1512-1515.

Maris E, Oostenveld R (2007) Nonparametric statistical testing of EEG- and MEG-data. J Neurosci Methods 164:177-190.

Medendorp WP, Kramer GF, Jensen O, Oostenveld R, Schoffelen JM, Fries P (2007) Oscillatory activity in human parietal and occipital cortex shows hemispheric lateralization and memory effects in a delayed double-step saccade task. Cereb Cortex 17:2364-2374.

Meltzer JA, Zaveri HP, Goncharova II, Distasio MM, Papademetris X, Spencer SS, Spencer DD, Constable RT (2008) Effects of working memory load on oscillatory power in human intracranial EEG. Cereb Cortex 18:1843-1855.

Miller EK, Desimone R (1994) Parallel neuronal mechanisms for shortterm memory. Science 263:520-522.

Mitra PP, Bokil H (2008) Observed brain dynamics. New York: Oxford UP.

Müri RM, Rivaud S, Vermersch AI, Léger JM, Pierrot-Deseilligny C (1995) Effects of transcranial magnetic stimulation over the region of the supplementary motor area during sequences of memory-guided saccades. Exp Brain Res 104:163-166.

Nolte G (2003) The magnetic lead field theorem in the quasi-static approximation and its use for magnetoencephalography forward calculation in realistic volume conductors. Phys Med Biol 48:3637-3652.

Onton J, Delorme A, Makeig S (2005) Frontal midline EEG dynamics during working memory. Neuroimage 27:341-356.

Oostenveld R, Fries P, Maris E, Schoffelen JM (2011) FieldTrip: Open source software for advanced analysis of MEG, EEG, and invasive electrophysiological data. Comput Intell Neurosci 2011:156869.

Palva JM, Monto S, Kulashekhar S, Palva S (2010) Neuronal synchrony reveals working memory networks and predicts individual memory capacity. Proc Natl Acad Sci U S A 107:7580-7585.

Palva S, Kulashekhar S, Hämäläinen M, Palva JM (2011) Localization of cortical phase and amplitude dynamics during visual working memory encoding and retention. J Neurosci 31:5013-5025.

Pesaran B, Pezaris JS, Sahani M, Mitra PP, Andersen RA (2002) Temporal structure in neuronal activity during working memory in macaque parietal cortex. Nat Neurosci 5:805-811.

Pfurtscheller G, Neuper C, Andrew C, Edlinger G (1997) Foot and hand area mu rhythms. Int J Psychophysiol 26:121-135.

Rainer G, Asaad WF, Miller EK (1998) Selective representation of relevant information by neurons in the primate prefrontal cortex. Nature 393:577-579.

Rouder JN, Morey RD, Morey CC, Cowan N (2011) How to measure working memory capacity in the change detection paradigm. Psychon Bull Rev 18:324-330.

Sauseng P, Klimesch W, Doppelmayr M, Pecherstorfer T, Freunberger R, Hanslmayr S (2005) EEG alpha synchronization and functional coupling during top-down processing in a working memory task. Hum Brain Mapp 26:148-155.

Sauseng P, Klimesch W, Heise KF, Gruber WR, Holz E, Karim AA, Glennon M, Gerloff C, Birbaumer N, Hummel FC (2009) Brain oscillatory substrates of visual short-term memory capacity. Curr Biol 19:1846-1852.

Stanislaw H, Todorov N (1999) Calculation of signal detection theory measures. Behav Res Methods Instrum Comput 31:137-149. 
Sumner P, Nachev P, Morris P, Peters AM, Jackson SR, Kennard C, Husain M (2007) Human medial frontal cortex mediates unconscious inhibition of voluntary action. Neuron 54:697-711.

Tallon-Baudry C, Bertrand O, Peronnet F, Pernier J (1998) Induced gamma-band activity during the delay of a visual short-term memory task in humans. J Neurosci 18:4244-4254.

Uhlhaas PJ, Singer W (2010) Abnormal neural oscillations and synchrony in schizophrenia. Nat Rev Neurosci 11:100-113.

Van Der Werf J, Jensen O, Fries P, Medendorp WP (2010) Neuronal synchronization in human posterior parietal cortex during reach planning. J Neurosci 30:1402-1412.

Vogel EK, McCollough AW, Machizawa MG (2005) Neural measures reveal individual differences in controlling access to working memory. Nature 438:500-503.

Wager TD, Smith EE (2003) Neuroimaging studies of working memory: a meta-analysis. Cogn Affect Behav Neurosci 3:255-274.

Worden MS, Foxe JJ, Wang N, Simpson GV (2000) Anticipatory biasing of visuospatial attention indexed by retinotopically specific alphaband electroencephalography increases over occipital cortex. J Neurosci 20:RC63.

Yamanaka K, Yamagata B, Tomioka H, Kawasaki S, Mimura M (2010) Transcranial magnetic stimulation of the parietal cortex facilitates spatial working memory: near-infrared spectroscopy study. Cereb Cortex 20: 1037-1045. 